Revista de Matemática: Teoría y Aplicaciones 2003 10(1-2) : 122-130

CIMPA - UCR - CCSS ISSN: 1409-2433

\title{
UN MÉTODO PARA LA CARACTERIZACIÓN EXHAUSTIVA DE FORMAS DE FIGURAS PLANAS, TANTO SIMPLE COMO MÚLTIPLEMENTE CONEXAS
}

\author{
Osvaldo Skliar* $^{*} \quad$ Víctor Medina $^{\dagger} \quad$ José S. PovedA ${ }^{\ddagger}$
}

Recibido: 9 Jun 2003

\begin{abstract}
Resumen
Se presenta un método que posibilita, mediante ciertos entes matemáticos descriptores obtenidos a partir de figuras planas -que pueden ser simple o múltiplemente conexas- una caracterización exhaustiva de las formas de dichas figuras. En efecto; a partir de esos entes matemáticos -que serán denominados "curvas características" de las correspondientes figuras- resulta factible reconstruir las figuras en cuestión. Una vez especificado qué se entenderá por "distancia" entre dos "pixels" cualesquiera, cada una de dichas curvas características es generada como función de los elementos pertenecientes a dos conjuntos de distancias entre determinados "pixels".

Las curvas características de las figuras consideradas resultan independientes de las posiciones de esas figuras respecto de cualquier sistema de referencia. En otras palabras, son invariantes al tener lugar desplazamientos y/o rotaciones cualesquiera de dichas figuras.
\end{abstract}

Palabras clave: caracterización de formas, contornos de figuras planas, método de triangulación, centro de masa, reconocimiento de patrones.

\begin{abstract}
This article presents a method with which the shapes of plane figures may be characterized exhaustively by using descriptive mathematical entities obtained from these figures. They may be either simply or multiply connected. Thus the particular figures may be reconstructed from these mathematical entities which will be known as the "characteristic curves" of the corresponding figures. Once what is understood by

\footnotetext{
*Escuela de Informática, Universidad Nacional, Heredia, Costa Rica. e-mail: oskliar@racsa.co.cr

${ }^{\dagger}$ Escuela de Matemática, Universidad Nacional, Heredia, Costa Rica. e-mail: vmedinab@racsa.co.cr

${ }^{\ddagger}$ Laboratorio de Matemática Aplicada y Simulación Computacional, Escuela de Matemática, Universidad Nacional, Heredia, Costa Rica. e-mail: seniormaster@hotmail.com
} 
the "distance" between any two pixels has been specified, each of these characteristic curves is generated as a function of the elements belonging to the two sets of distances between particular pixels.

The characteristic curves of the figures considered are independent of the positions of these figures with regard to any reference system. In other words, they are invariable even when the figures are subjected to displacements or rotations.

Keywords: shape characterization, contours of plane figures, triangulation method, center of mass, pattern recognition

Mathematics Subject Classification: 68T10

\section{Introducción}

Actualmente, una de las áreas más cultivadas -y en la que mayor dinamismo se puede advertir- del vasto campo del reconocimiento de patrones - "pattern recognition" - es la orientada a la caracterización y análisis de formas de objetos -tanto de dos como de tres dimensiones- y a la clasificación de dichos objetos según sus formas [1].

El objetivo de este trabajo es presentar un método para la caracterización exhaustiva de formas de objetos planos ${ }^{1}$, tanto simple como múltiplemente conexos. En lo sucesivo, se denominará "método de triangulación" dicho método, por razones que resultarán claras al describirlo.

Sea $\mathcal{O}$ un objeto plano. Si $\mathcal{O}$ es simplemente conexo sólo tendrá un contorno $\mathcal{C}$. Si $\mathcal{O}$ es múltiplemente conexo tendrá, además de un contorno externo $\mathcal{C}$, cierto número de contornos "internos" $-\mathcal{C}_{1}, \mathcal{C}_{2}, \mathcal{C}_{3}, \ldots, \mathcal{C}_{n}-$. El método de triangulación permitirá codificar mediante una "curva característica" $-\mathcal{C C}$ - cada uno de los contornos de $\mathcal{O}$. Si $\mathcal{O}$ tiene más de un contorno -o sea, cuenta con los contornos $\mathcal{C}, \mathcal{C}_{1}, \mathcal{C}_{2}, \ldots, \mathcal{C}_{n^{-}}$las correspondientes curvas características serán denominadas $\mathcal{C C}, \mathcal{C C}_{1}, \mathcal{C C}_{2}, \ldots, \mathcal{C C}_{n}$. Dichas curvas características resultan, en gran medida, invariantes respecto de translaciones y rotaciones cualesquiera de $\mathcal{O}$. Las ligeras variaciones que se puede advertir en las curvas características al transladar o rotar $\mathcal{O}$ son debidas a que, en muchos casos, $\mathcal{O}$ mismo experimenta ligeros cambios al ser desplazado o rotado. ¿. Por qué? Porque $\mathcal{O}$ es un objeto "digitalizado". A título de ejemplo, en la figura 1 puede notarse el cambio experimentado por una región del contorno de un rectángulo digitalizado al ser rotado $60^{\circ}$ en sentido antihorario.

\section{Descripción del método}

\subsection{Aplicación del método de triangulación para caracterización de for- mas de objetos planos simplemente conexos}

Para cada objeto considerado, se determina, en primer lugar, el centro de masa (CM) de dicho objeto. Sean $p_{1}, p_{2}, \ldots, p_{n}$ los pixels constitutivos de un dado objeto $\mathcal{O}$. Admítase

\footnotetext{
${ }^{1}$ En este artículo, la expresión "objeto plano" será considerada sinónimo de la expresión "figura plana".
} 

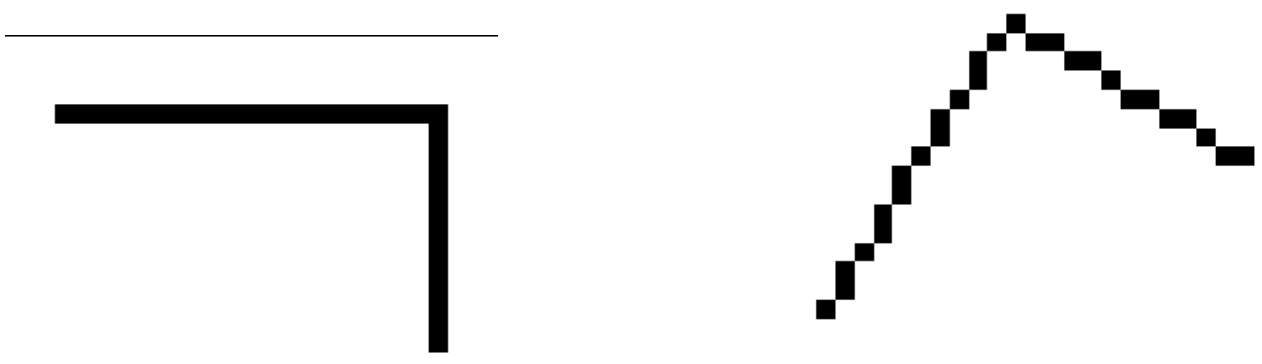

1.a) $\mathcal{O}$ es una región del contorno de un $60^{\circ}$ en sentido antihorario rectángulo digitalizado

1.b) $\mathcal{O}^{\prime}$ es el objeto obtenido al $\operatorname{rotar} \mathcal{O}$

\section{Figura 1}

que las coordenadas correspondientes a cada uno de dichos pixels son $\left(x_{1}, y_{1}\right),\left(x_{2}, y_{2}\right)$, $\ldots,\left(x_{n}, y_{n}\right)$. Sea $m$ la "masa" que se atribuye a cada uno de esos pixels. Entonces, las coordenadas del centro de masa $-\left(x_{\mathrm{CM}}, y_{\mathrm{CM}}\right)-$ son:

$$
x_{\mathrm{CM}}=\frac{\sum_{i=1}^{n} m \cdot x_{i}}{\sum_{i=1}^{n} m}, \quad y_{\mathrm{CM}}=\frac{\sum_{i=1}^{n} m \cdot y_{i}}{\sum_{i=1}^{n} m}
$$

Admítase que la masa correspondiente a cada pixel es numéricamente igual a 1. Entonces, las coordenadas del centro de masa del objeto pueden ser expresadas así:

$$
x_{\mathrm{CM}}=\frac{\sum_{i=1}^{n} x_{i}}{n}, \quad y_{\mathrm{CM}}=\frac{\sum_{i=1}^{n} y_{i}}{n}
$$

Son determinadas, también, las coordenadas de aquel pixel del contorno externo del objeto que está más alejado del centro de masa. O sea, las coordenadas del pixel del contorno externo cuya distancia al centro de masa es la mayor de todas las distancias entre cada uno de los pixels del contorno y el centro de masa. Dicho pixel se denominará $P_{\text {ext }^{2}}$.

\footnotetext{
${ }^{2}$ Para el caso en que cada uno de dos o más pixels del contorno estén a una distancia igual a la distancia máxima a la que se hizo referencia, se desarrolló un algoritmo que permite a) determinar cuál de esos pixels debe ser considerado $P_{\text {ext }}$, o bien b) concluir que resulta indiferente considerar $P_{\text {ext }}$ cualquiera de esos pixels. (Dicho algoritmo no será especificado en este artículo.)
} 
Los pixels del contorno del objeto son numerados, a partir del pixel $P_{\text {ext }}$, inclusive, -al que se le hace corresponder el número 1-recorriendo el contorno en sentido horario. Para cada uno de los pixels constitutivos del contorno externo del objeto $-p_{i, \text { ext }}(i=$ $1,2, \ldots, l)$ - son computadas dos distancias:

a) la distancia $d_{i, 1}(i=1,2, \ldots, l)$, existente entre $p_{i, \text { ext }}$ y el centro de masa, y

b) la distancia $d_{i, 2}(i=1,2, \ldots, l)$, existente entre $p_{i, \text { ext }}$ y $P_{\text {ext }}$. (Dado el criterio elegido para la numeración de los pixels del contorno externo del objeto, es obvio que $d_{1,2}$ es, en todos los casos, igual a cero.)

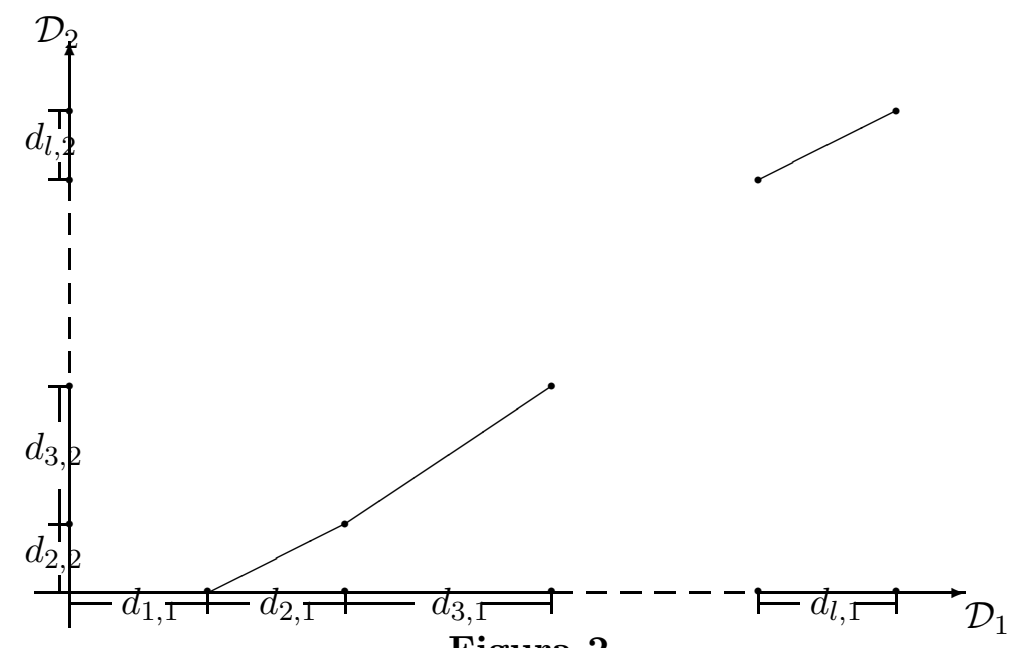

Figura 2

La figura 2 ilustra cómo se construye la curva característica correspondiente al contorno externo de $\mathcal{O}$.

Los puntos destacados en el eje de las abscisas $-\mathcal{D}_{1}$ - representan las sumas acumuladas de las distancias $d_{1,1}, d_{2,1}, \ldots y d_{l, 1}$. En otras palabras, dichos puntos corresponden a las sumas acumuladas $d_{1,1}, d_{1,1}+d_{2,1}, d_{1,1}+d_{2,1}+d_{3,1}, \ldots$ y $d_{1,1}+d_{2,1}+d_{3,1}+\cdots+d_{l, 1}$. Los puntos destacados en el eje de las ordenadas $-\mathcal{D}_{2}$ - representan las sumas acumuladas de las distancias $d_{1,2}, d_{2,2}, \ldots y d_{l, 2}$. En otras palabras, dichos puntos corresponden a las sumas acumuladas $d_{1,2}, d_{1,2}+d_{2,2}, d_{1,2}+d_{2,2}+d_{3,2}, \ldots$ y $d_{1,2}+d_{2,2}+d_{3,2}+\cdots+d_{l, 2}$.

Es obvio que a partir de la curva característica $-\mathrm{CC}$ - correspondiente a un objeto plano simplemente conexo puede recontruirse dicho objeto.

Sean los objetos planos representados en la figura 3.

La figura $3 \mathrm{~b}$ representa el objeto que se obtiene sometiendo el objeto representado en la figura 3a a una rotación de $45^{\circ}$ en sentido antihorario. Las curvas características correspndientes a dichos objetos se han represntado en la figura 4. 


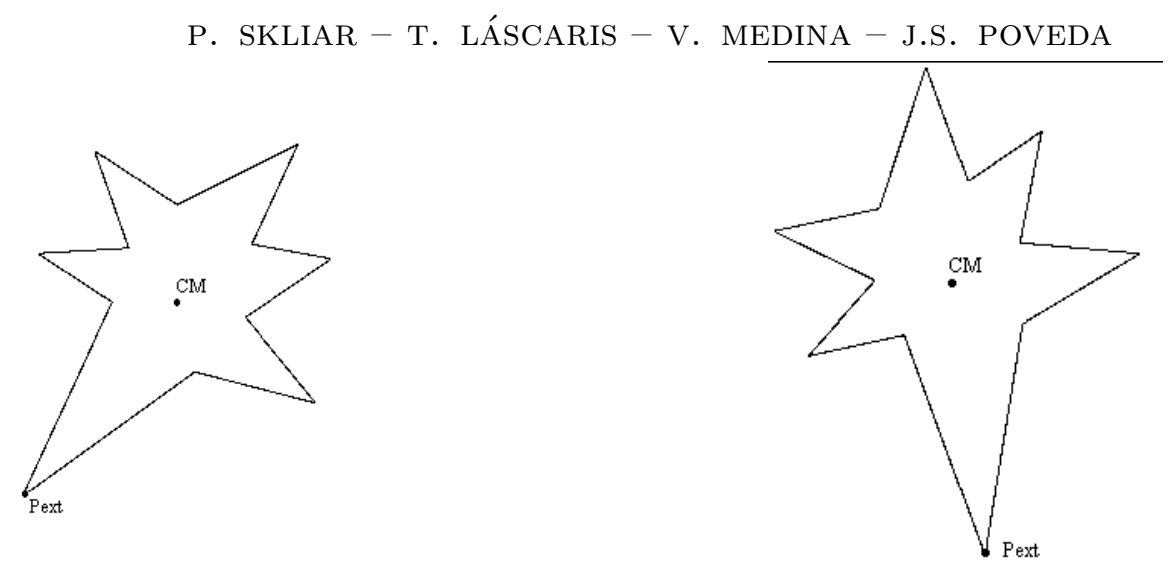

Figura 3.a)

Figura 3.b)

\section{Figura 3}

\subsection{Aplicación del método de triangulación para caracterización de for- mas de objetos planos múltiplemente conexos}

Supóngase que el objeto plano múltiplemente conexo cuenta no sólo con un contorno externo sino también con $k$ contornos internos. En este caso, convendrá numerar dichos contornos internos para poder hacer referencia, sin ambiguedad, a cualquiera de ellos. Así, podrá distinguirse entre el "contorno interno 1", el "contorno interno 2", etc..

La curva característica correspondiente al contorno externo del objeto se obtiene de la manera descripta en el apartado 2.1.

A título de ejemplo de la forma en que se procede para obtener las curvas características correspondientes a los contornos internos, se especificará la forma de obtener la curva característica correspondiente al contorno interno 1. Son determinadas las coordenadas de aquel pixel del contorno interno 1 que está más alejado del CM. (O sea, las coordenadas del pixel del contorno interno 1 cuya distancia al CM es la mayor de todas las distancias entre cada uno de los pixels de dicho contorno interno y el CM.) Ese pixel se denomina $P_{\text {int,1. }}$. (En el caso de que cada uno de dos o más pixels del contorno interno 1 estén a una distancia igual a la distancia máxima a la que se hizo referencia, se selecciona, entre aquelos pixels, el pixel que se denomina $P_{\text {int, } 1}$, mediante la utilización del algoritmo mencionado previamente, en una nota a pie de página.)

Los pixels del contorno interno 1 del objeto son numerados, a partir de $P_{\text {int, } 1}$, inclusive, recorriendo dicho contorno en sentido horario. Luego -utilizando CM, $P_{\text {ext }}$ y el conjunto numerado de pixels del contorno interno 1- se obtiene la curva característica correspondiente al contorno interno 1 , mediante el mismo procedimiento que condujo a la obtención de la curva característica correspondiente al contorno externo -utilizando CM, $P_{\text {ext }}$ y el conjunto numerado de pixels del contorno externo--

En la figura 5 se ha representado un objeto que cuenta no sólo con un contorno externo sino también con dos contornos internos.

En las figuras 6, 7 y 8 se han representado las curvas características correspondientes al 


\section{Curvas carcterísticas correspondientes a los obj etos de la Figura 3}

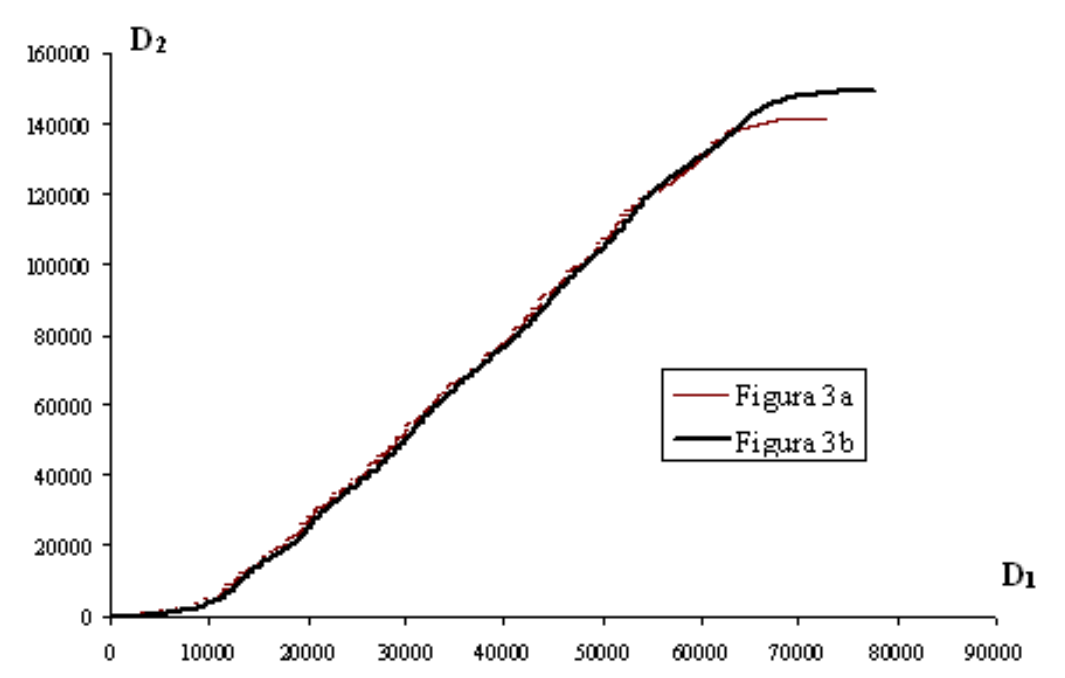

Figura 4

contorno externo y a los contornos internos 1 y 2 , respectivamente, del objeto representado en la figura 5.

A partir de estas tres curvas características, puede reobtenerse el objeto representado en la figura 5. El objeto recuperado se muestra en la figura 9.

\section{$3 \quad$ Perspectivas}

En este artículo se han presentado, de manera escueta y preliminar, las ideas fundamentales de un nuevo método - el método de triangulación- para la caracterización de formas de objetos planos, tanto simple como múltiplemente conexos. Si bien otros enfoques son superiores al descripto para alcanzar objetivos como caracterizar diversas zonas de los contornos de los objetos según características tales como, p.ej., sus grados de convexidad o concavidad ([2], [3]), el método introducido en este trabajo es superior a la mayoría de los métodos disponibles en cuanto a la completitud de la caracterización que posibilita de los objetos considerados. Pero, en opinión de los autores, la principal ventaja del método de triangulación radica en las posibilidades que brinda para tareas de clasificación de objetos según sus formas. A este último asunto será dedicado otro artículo.

\section{Referencias}

[1 ] da Fontoura Costa, L.; Marconde Cesar Jr., R. (2001) Shape Analysis and Classification: Theory and Practice. CRC Press, Washington, D.C. 


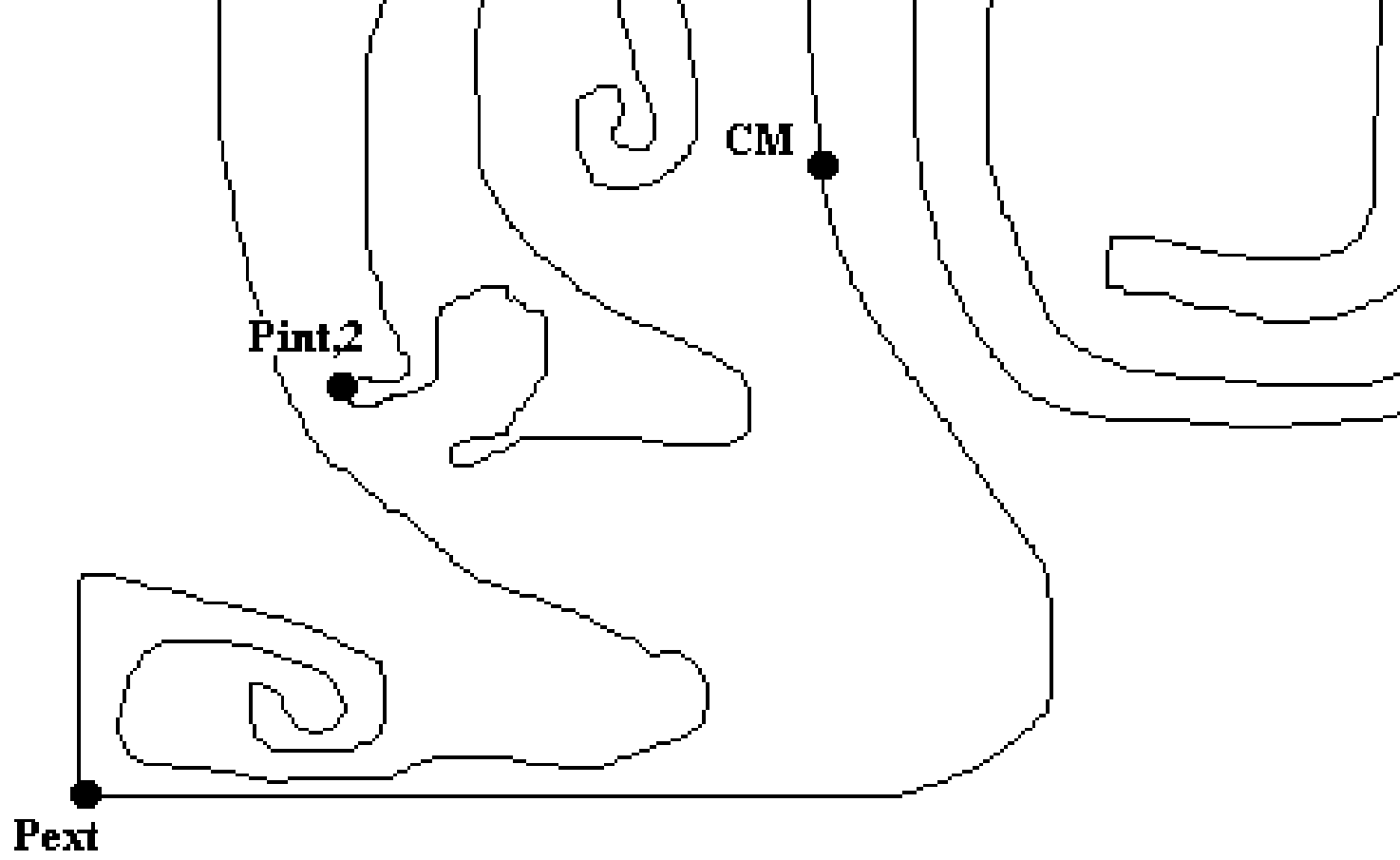

Figura 5

[2 ] Skliar, O.; Loew, M.H. (1985) "A new method for characterization of shape", Pattern Recognition Letters, vol. 3(5): pp. 335-342.

[3 ] Skliar, O.; Láscaris Comneno, T.; Medina, V.; Poveda, J. (2002) "Una variante del método que utiliza la simulación de un proceso de difusión para la caracterización de formas de figuras planas", Revista de Matemática: Teoría y Aplicaciones, publicado en este mismo número. 
Curva carac terística correspondiente al contorno externo del objeto de la Figura 5

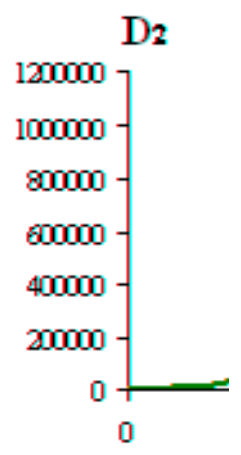

\section{Curva carac teristica correspo al contomo interno $l$ del obje Figura 5}

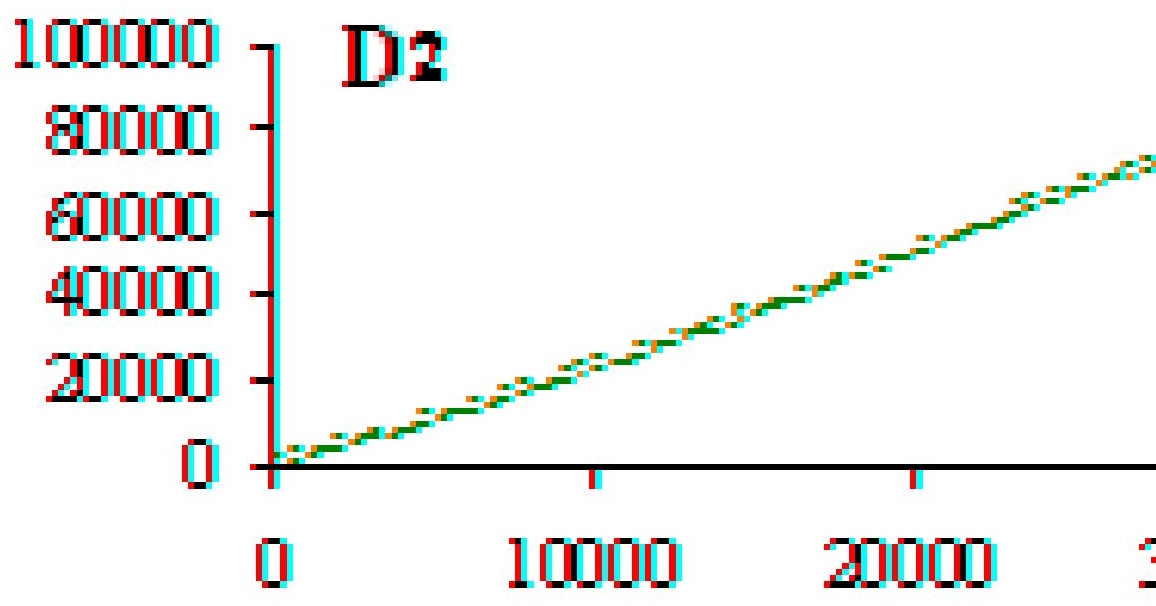

Figura 7 


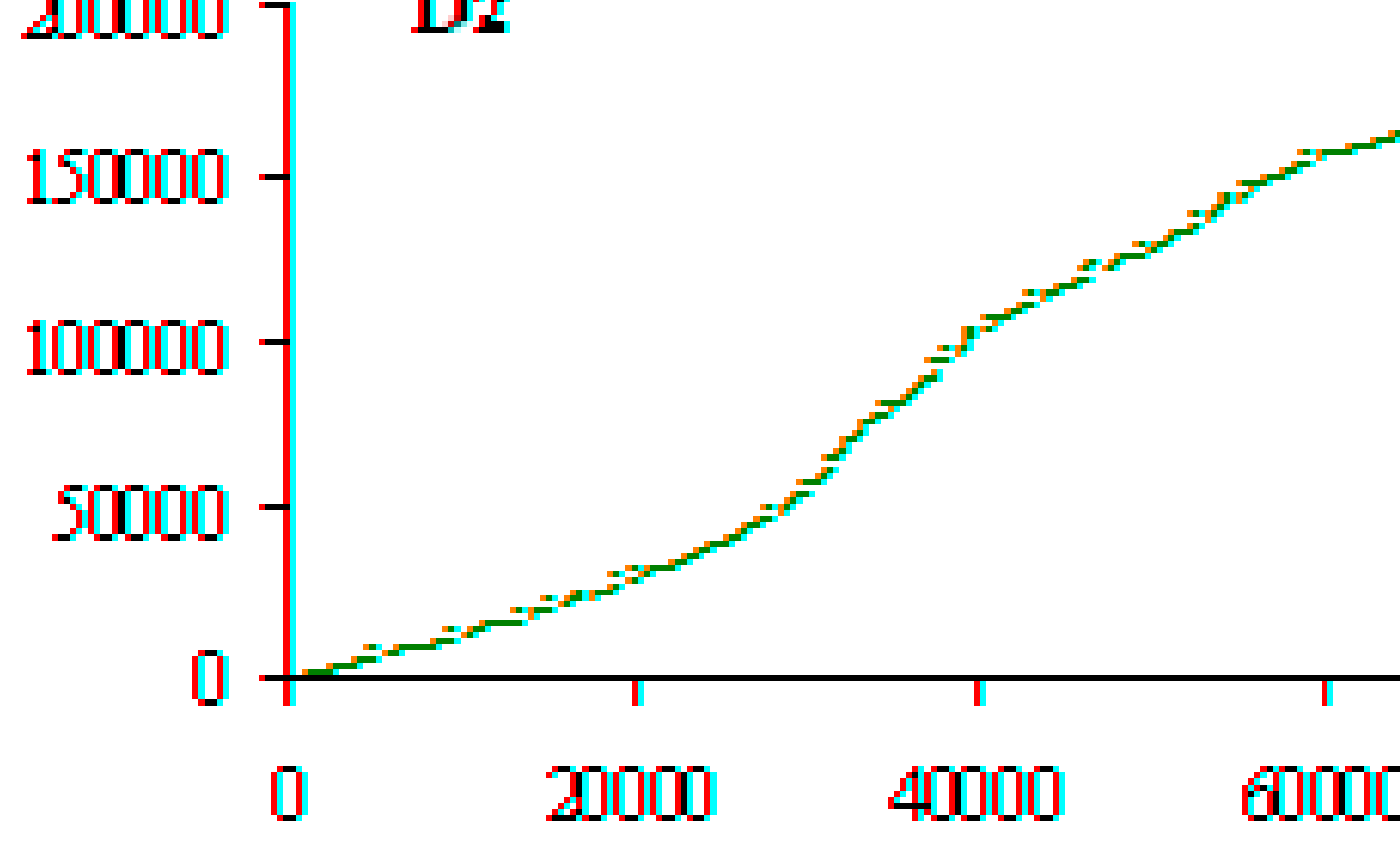

Figura 8

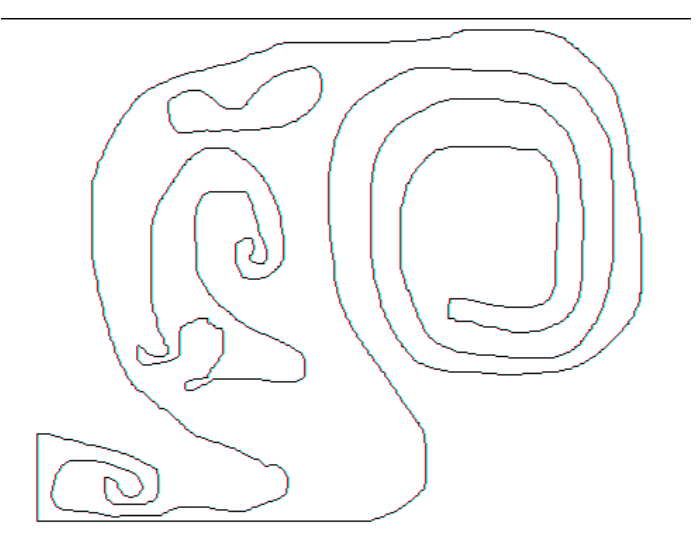

Figura 9 